\begin{abstract}
PHASKLIS
Disiplinlerarası Akdeniz Araştırmaları Dergisi

Journal of Interdisciplinary Mediterranean Studies

journal.phaselis.org

Volume II (2016)

open $\bigcirc$ access journals

PHASELIS: Disiplinlerarası Akdeniz Araştırmaları Dergisi'nde bulunan içeriklerin tümü kullanıcılara açık, serbestçe/ücretsiz "açık erişimli" bir dergidir. Kullanıcılar, yayıncıdan ve yazar(lar)dan izin almaksızın, dergideki makaleleri tam metin olarak okuyabilir, indirebilir, dağıtabilir, makalelerin çıktısını alabilir ve kaynak göstererek makalelere bağlantı verebilir.

PHASELIS: Disiplinlerarası Akdeniz Araştırmaları Dergisi uluslararası hakemli elektronik (online) bir dergi olup değerlendirme süreci biten makaleler derginin web sitesinde (journal.phaselis.org) yıl boyunca ilgili sayının içinde (Volume II: Ocak-Aralık 2016) yayımlanır. Aralık ayı sonunda ilgili yıla ait sayı tamamlanır.

Dergide yayımlanan eserlerin sorumluluğu yazarlarına aittir.
\end{abstract}

Atıf Düzeni M. S. Taylan - D. Şirin, "Phaselis Antik Kenti Orthoptera (Insecta) Faunası I". Phaselis II (2016) 49-56. DOI: 10.18367/Pha.16004

Geliş Tarihi: 10.03.2016 | Kabul Tarihi: 05.05.2016 | Online Yayın Tarihi: 24.05.2016

Editörya Phaselis Research Project

www.phaselis.org 


\title{
Phaselis Antik Kenti Orthoptera (Insecta) Faunası I
}

\section{Orthoptera (Insecta) Fauna of the Ancient City of Phaselis I}

\author{
M. Sait TAYLAN* Deniz ŞiRIN**
}

\begin{abstract}
Öz: Bu çalışmada, Phaselis Antik Kenti Orthoptera (Insecta) faunası çalışmalarının ilk bölümü olan, tümü yazarlar tarafından 2015 yılında toplanan ve Namık Kemal Üniversitesi Entomoloji Müzesinde muhafaza edilen örneklerin taksonomik değerlendirmeleri ve tür listesi sunulmuştur. Yapılan ilk araştırmalar sonucunda, Phaselis Antik Kenti'nde yayılış gösteren Orthoptera takımının, 2 alttakım, 6 familya, 15 altfamilya, 23 cinse ait 27 tür/alttür ile temsil edildiği ortaya çıkarılmışıı. Tespit edilen taksonların tümü Phaselis Antik Kenti için yeni kayıt olup, tarımsal ve ekili alanlara zarar verdikleri bilinen 11 tür tespit edilmiştir.
\end{abstract}

Anahtar Sözcükler: Orthoptera · Fauna $\cdot$ Caelifera $\cdot$ Ensifera $\cdot$ Phaselis

Abstract: In this study the species list and the taxonomic evaluation of the Orthoptera (Insecta) fauna found in the ancient city of Phaselis Ancient City, is presented through the specimens which collected in 2015 by the authors and kept in Namık Kemal University Entomology Museum. The results of this preliminary survey show the Orthoptera fauna of Phaselis consists of, 2 suborders, 6 families, 15 subfamilies and 27 species/subspecies belonging to 23 genus. All the identified taxa are new records for the ancient city of Phaselis, while 11 of these species are known to cause damage to agricultural and cultivated areas.

Keywords: Orthoptera $\cdot$ Fauna $\cdot$ Caelifera $\cdot$ Ensifera $\cdot$ Phaselis

\section{Giriş}

Anadolu, buzul dönemlerde farklı formlar için sığınak görevi görmesi, üç fitocoğrafik bölgeden elemanlar barındırması, iklim ve ekosistem çeşitliliği, jeolojik, jeomorfolojik ve topoğrafik çeşitliliklere sahip olması nedeniyle biyoçeşitliliği oldukça yüksek bir kara parçasıdır. Milli parklar da bu zengin biyoçeşitliliğin en doğal olarak gözlendiği ve korunduğu alanların başında gelmektedir. Çalışma alanı olan Phaselis Antik Kenti, Antalya ilii Olympos Beydağları Milli Parkı sınırları içerisinde olup, deniz, orman ve dağ ekosistemi gibi birçok ekosistemi barındıran bir milli park olmasıyla ayrı bir öneme sahiptir.

Faunistik çalışmamıza konu olan Orthoptera (çekirgeler) takımı, Caelifera (kısa antenli çekirgeler) ve Ensifera (uzun antenli çekirgeler) olmak üzere iki alttakımdan oluşmaktadır. Bu alttakımlardan, Caelifera alttakımı Türkiye'de, Acrididae familyasından 176, Pamphagidae familyasından 66, Tetrigidae familyasından 9, Pyrgomorphidae familyasından 5 ve Tridactylidae

* Dr., Anadolu Speleoloji Grubu Derneği, İstanbul. msaittaylan@yahoo.com

** Doç. Dr., Namık Kemal Üniversitesi, Fen Edebiyat Fakültesi, Biyoloji Bölümü, Tekirdağ. denizsirin19@gmail.com 
familyasından 4 takson ile temsil edilmektedir ${ }^{1}$. Ensifera alttakımı ise ülkemizde 6 familya içermektedir. Tettigoniidae familyası en çok takson içeren familya olup, neredeyse alttakımın sahip olduğu taksonların yarısı bu familyaya dâhildir. Diğer taraftan Gryllidae 36, Gryllotalpidae 36, Rhaphidophoridae 17, Mogoplistidae 4, Schizodactylidae ve Gryllotalpidae ise birer takson ile temsil edilmektedir² .

Orthoptera takımı, diğer böcek gruplarına göre nispeten iyi çalışılmış gruplardan biridir. Şimdiye kadar, Türkiye Orthoptera faunasıyla ilgili fazla sayıda çalışma mevcuttur. Ancak şu ana kadar çalışma alanımız olan Phaselis Antik Kentinde Orthoptera faunasıyla ilgili yapılmış kapsamlı bir çalışma mevcut değildir. Ancak yakın alanlarda bazı çalışmalar mevcuttur. Bu çalışmalara ${ }^{3}$ belirtilen dipnottaki kaynaklar örnek olarak verilebilir.

Bu çalışmada amacımız, Orthoptera (Insecta) faunası henüz çalışımamış olan Phaselis Antik Kenti'nin Orthoptera faunasının ortaya çıkarılmasıdır.

\section{Materyal ve Metot}

Bu çalışmada 2015 yılında, Phaselis Antik Kenti'nde tamamı yazarlar tarafından toplanan örnekler incelenmiştir. Örnekler atrap ile toplanmış, \%70'lik etanol içeren tüplere alınıp laboratuvara getirilmiş ve standart yöntemlerle preparasyonları yapılarak müze materyali haline getirilmiştir. Preparasyonu yapılan örnekler Leica Z6 APO, Leica EZ4D ve Optica SZM-SMD ışık mikroskoplarıyla incelenmiştir. Örneklerin teşhis edilmesinde ${ }^{4}$ (belirtilen dipnottaki yazarlar) tarafından yapılan çalışmalar izlenmiştir. İncelenen örnekler Namık Kemal Üniversitesi, Biyoloji Bölümü, Entomoloji Müzesi'nde (NKUEM - Tekirdağ/Türkiye) muhafaza edilmektedir.

\section{Bulgular}

Yapılan incelemeler sonucunda, Phaselis Antik Kenti'nde yayılış gösteren Orthoptera takımının, 2 alttakım, 6 familya, 15 altfamilya, 23 cinse ait 27 tür/alttür ile temsil edildiği ortaya çıkarılmış ve bu taksonların isimleri ve ait olduğu alttakım, familya, altcins ve cins bilgileri aşağıda liste halinde sunulmuştur.

\section{ORDO: ORTHOPTERA}

\section{SUBORDO: CAELIFERA}

\subsection{FAMILY: ACRIDIDAE MaCLEAY, 1821}

1.1.1. SUBFAMILY: OEDIPODINAE WALKER, 1871

1.1.1.1. Genus: Acrotylus Fieber, 1853

Acrotylus insubricus insubricus (Scopoli, 1788) (Fig. 1).

1.1.1.2. Genus: Aiolopus Fieber, 1853

Aiolopus strepens strepens (Latreille, 1804)

(Fig.2).
1.1.1.3. Genus: Oedipoda Latreille, 1829 Oedipoda aurea Uvarov, 1923 (Fig. 3). Oedipoda miniata miniata (Pallas, 1771) (Fig. 4). Oedipoda coerulescens (Linnaeus, 1758)

1.1.1.4. Genus: Locusta Linnaeus, 1758 Locusta migratoria migratoria (Linnaeus, 1758) (Fig. 5).

\subsubsection{SUBFAMILY: CALLIPTAMINAE TINKHAM,} 1940

\footnotetext{
Eades et al. 2016.

Ünal 2011.

Salman 1981; Güneş 1984; Naskrecki - Ünal 1995; Önder et al. 1999; Ünal 1999; 2001; Yalım - Çıplak 2002; Şirin et al. 2010; Tazegül - Önder 2012; Mol et al. 2014.

4 Uvarov 1934; Bei-Bienko - Mistshenko 1951; Ramme 1939; 1951; Karabağ 1956; 1957; 1958; Bei-Bienko 1965; Weidner 1969; Harz 1969; 1975; Demirsoy 1975; 1977; 1999²; Salman 1978; Soltani 1978; Güneş 1984; Willemse 1984; 1985; Naskrecki - Ünal 1995; Çıplak - Demirsoy 1996; Ünal 1999; 2011; Çıplak et al. 1999; Şirin et al. 2010; Sevgili et al. 2011; Eades et al. 2016.
} 


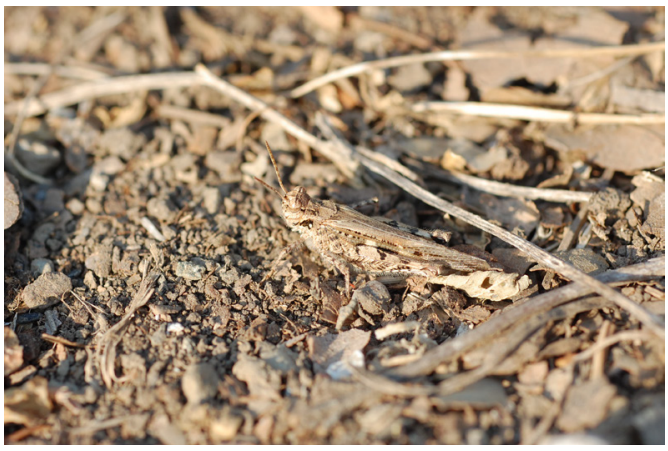

Fig. 1. Acrotylus insubricus insubricus (Scopoli, 1788)

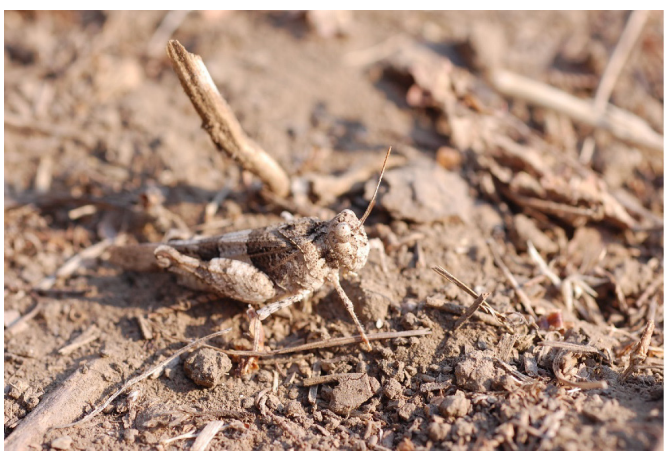

Fig. 3. Oedipoda aurea (Uvarov, 1923)

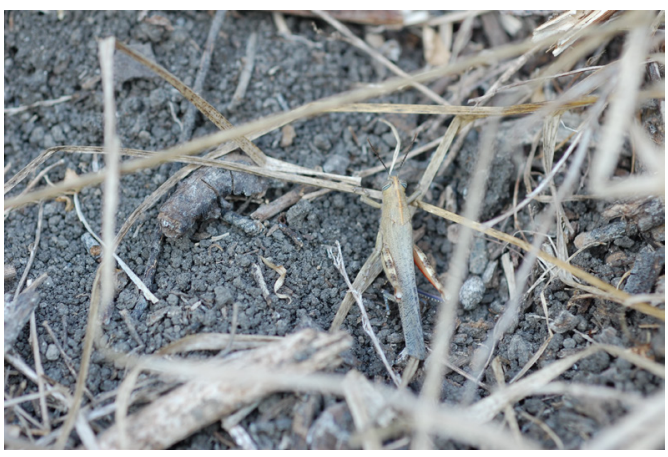

Fig. 5. Locusta migratoria migratoria (Linnaeus, 1758)

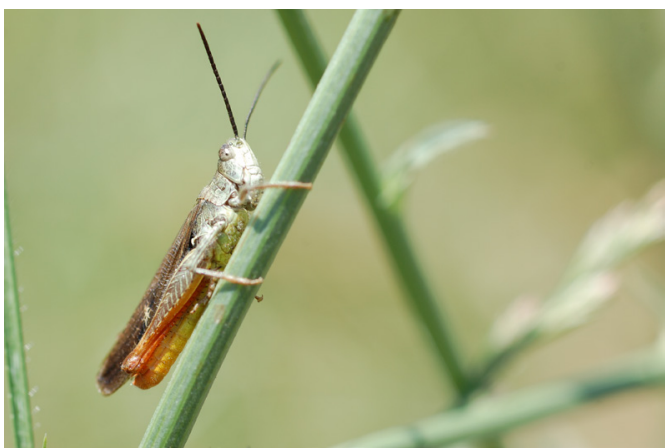

Fig. 7. Chorthippus (Glyptobothrus) vagans dissimilis (Willemse, Helversen et Odé, 2009)

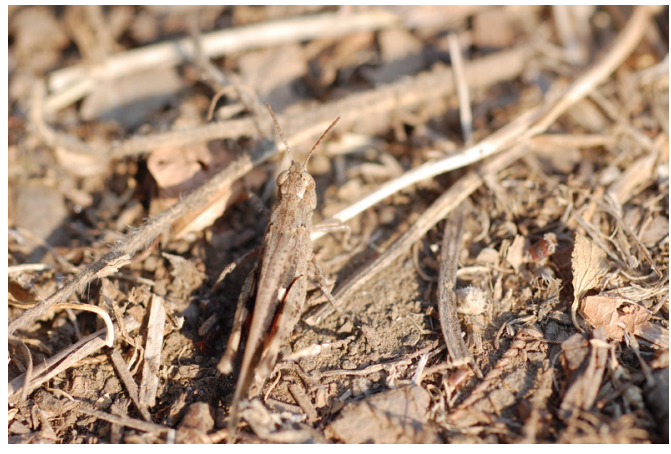

Fig. 2. Aiolopus strepens strepens (Latreille, 1804)

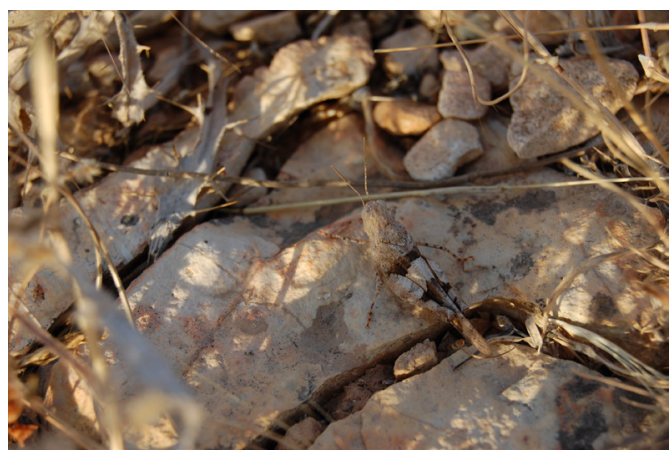

Fig. 4. Oedipoda miniata miniata (Pallas, 1771)

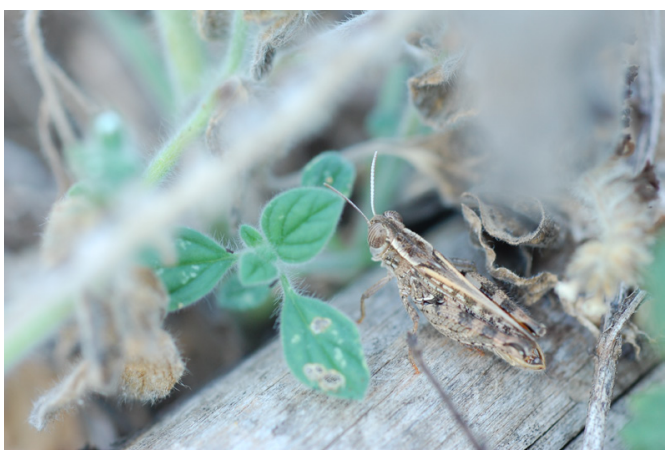

Fig. 6. Calliptamus italicus italicus (Linnaeus, 1758)

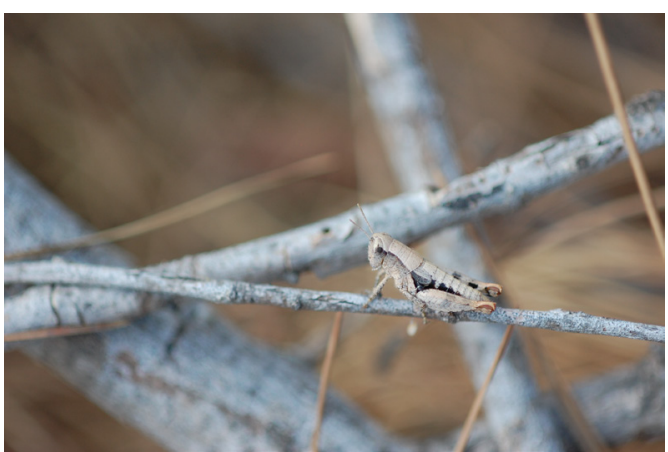

Fig. 8. Pezotettix giornae (Rossi, 1794) 
1.1.2.1. Genus: Calliptamus Serville, 1831

Calliptamus italicus italicus (Linnaeus, 1758)(Fig. 6).

1.1.3. SUBFAMILY: GOMPHOCERINAE FIEBER, 1853

1.1.3.1. Genus: Chorthippus Fieber, 1852 Chorthippus (Glyptobothrus) vagans dissimilis Willemse, Helversen et Odé, 2009 (Fig. 7).

1.1.3.2. Genus: Ramburiella Bolivar, 1906 Ramburiella (Palaeocesa) turcomana (Fischer von Waldheim, 1833)

1.1.3.3. Genus: Notostaurus Bey-Bienko,1933 Notostaurus anatolicus (Krauss, 1896) 1.1.4. SUBFAMILY: CATANTOPINAE BRUNNER VON WATTENWYL, 1893

1.1.4.1. Genus: Pezottetix Burmeister, 1840

Pezotettix giornae (Rossi, 1794) (Fig. 8).

1.1.5. SUBFAMILY: EYPREPOCNEMIDINAE BRUNNER VON WATTENWYL, 1893

1.1.5.1. Genus: Eyprepocnemis Fieber, 1853

Eyprepocnemis plorans plorans (Charpentier, 1825)(Fig. 9).

1.1.6. SUBFAMILY: CRYTACANTHACARIDINAE KIRBY, 1910

1.1.6.1. Genus: Anacridium Uvarov, 1923

Anacridium aegyptium (Linnaeus, 1764)

1.1.7. SUBFAMILY:

TROPIDOPOLINAE JACOBSON,1905

1.1.7.1. Genus: Tropidopola Stål, 1873

Tropidopola longicornis (Fieber, 1853)

1.2. FAMILY: PAMPHAGIDAE BURMEISTER, 1840

1.2.1. SUBFAMILY: ORCHAMINAE ZHANG, YIN \& YIN, 2003

1.2.1.1. Genus: Orchamus Stål, 1876

Orchamus davisi Uvarov, 1949 (Fig. 10)

1.3. FAMILY: TETRIGIDAE RAMBUR, 1838

1.3.1. SUBFAMILY: TETRIGINAE RAMBUR, 1838

1.3.1.1. Genus: Tetrix Latreille, 1802

Tetrix depressa Brisout de Barneville, 1848

\section{SUBORDO: ENSIFERA}

2.1.FAMILY: TETTIGONIIDAE KRAUSS, 1902

2.1.1. SUBFAMILY: TETTIGONIINAE KRAUSS, 1902

2.1.1.1. Genus: Eupholidoptera Maran, 1953 Eupholidoptera krueperi (Ramme, 1930) (Fig. 11).

2.1.1.2. Genus: Bucephaloptera Ebner, 1923

Bucephaloptera bucephala (Brunner von

Wattenwyl, 1882)

2.1.1.3. Genus: Decticus Serville, 1831

Decticus albifrons (Fabricius, 1775)

2.1.2. SUBFAMILY: PHANEROPTERINAE

BURMEISTER, 1838

2.1.2.1. Genus: Acrometopa Fieber, 1853

Acrometopa servillea (Brullé, 1832)(Fig. 12).

2.1.2.2. Genus: Poecilimon Fischer, 1853

Poecilimon (Poecilimon) luschani birandi Karaba, 1950 (Fig. 13).

2.1.2.3. Genus: Tylopsis Fieber, 1853

Tylopsis lilifolia (Fabricius, 1793)

2.1.3. SUBFAMILY: SAGINAE BRUNNER VON

WATTENWYL, 1878

2.1.3.1. Genus: Saga Charpentier, 1825

Saga rhodiensis Salfi, 1929 (Fig. 14).

2.2.FAMILY: GRYLLIIDAE LAICHARTING, 1781

2.2.1. SUBFAMILY: GRYLLINAE LAICHARTING, 1781

2.2.1.1.Genus:Gryllus Linnaeus, 1758

Gryllus (Gryllus) bimaculatus De Geer, 1773

2.2.1.2. Genus: Acheta Fabricius, 1775

Acheta domesticus (Linnaeus, 1758)

2.2.2. SUBFAMILY: OECANTHINAE BLANCHARD, 1845

2.2.2.1. Genus: Oecanthus Serville, 1831

Oecanthus pellucens pellucens (Scopoli, 1763)

2.3.FAMILY: GRYLLOTALPIDAE LEACH, 1815

2.3.1. SUBFAMILY: GRYLLOTALPINAE LEACH, 1815

2.3.1.1. Genus: Gryllotalpa Latreille, 1802

Gryllotalpa gryllotalpa (Linnaeus, 1758) 


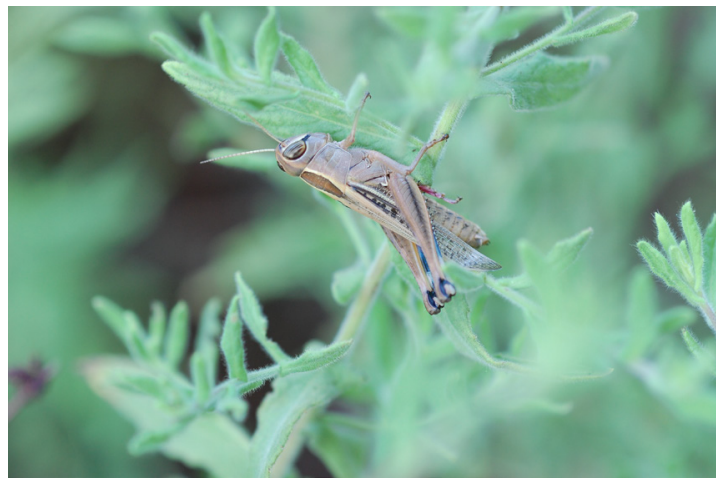

Fig. 9. Eyprepocnemis plorans plorans (Charpentier, 1825)

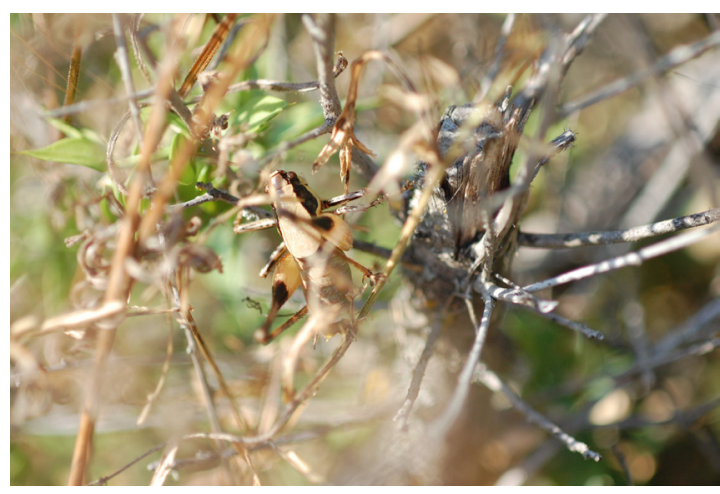

Fig. 11. Eupholidoptera krueperi (Ramme, 1930)

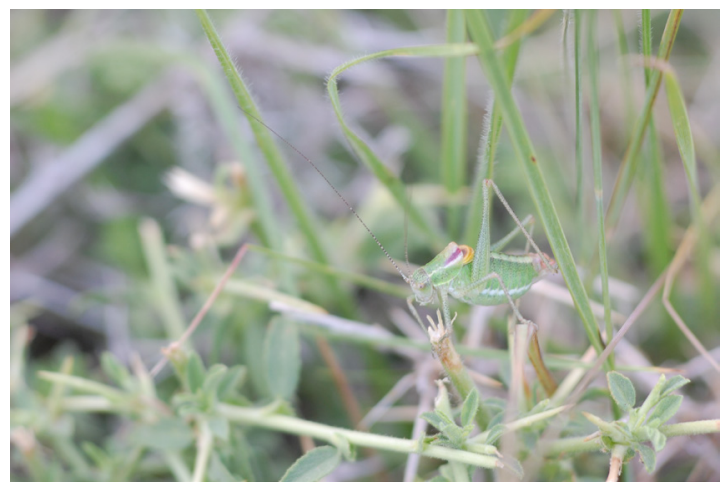

Fig. 13. Poecilimon (Poecilimon) luschani birandi (Karabag, 1950)

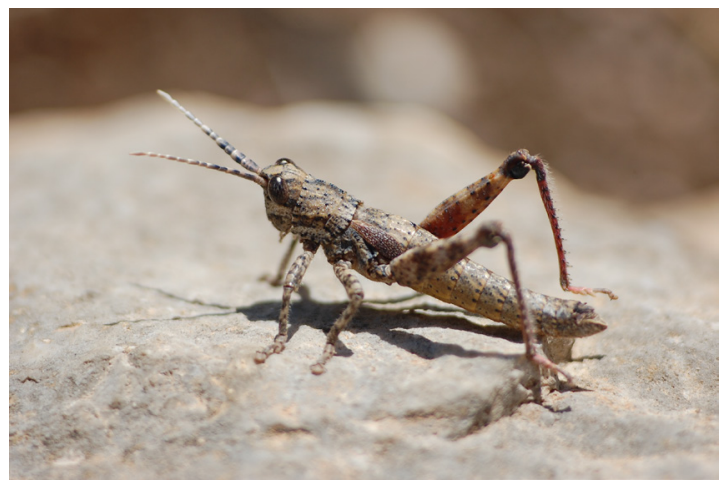

Fig. 10. Orchamus davisi (Uvarov, 1949)

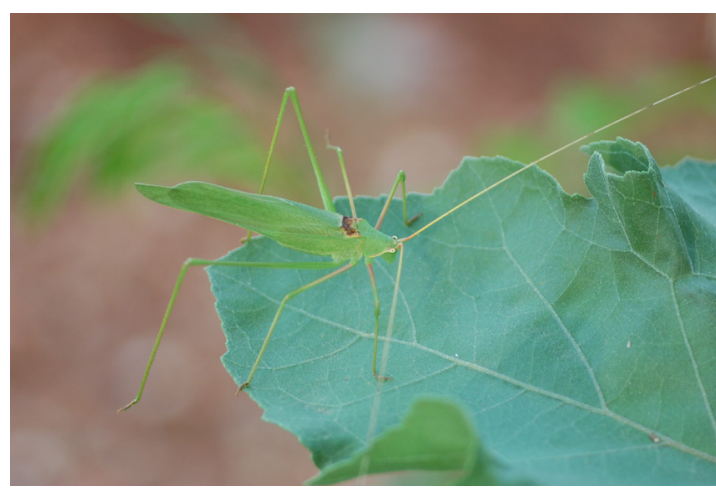

Fig. 12. Acrometopa servillea (Brullé, 1832)

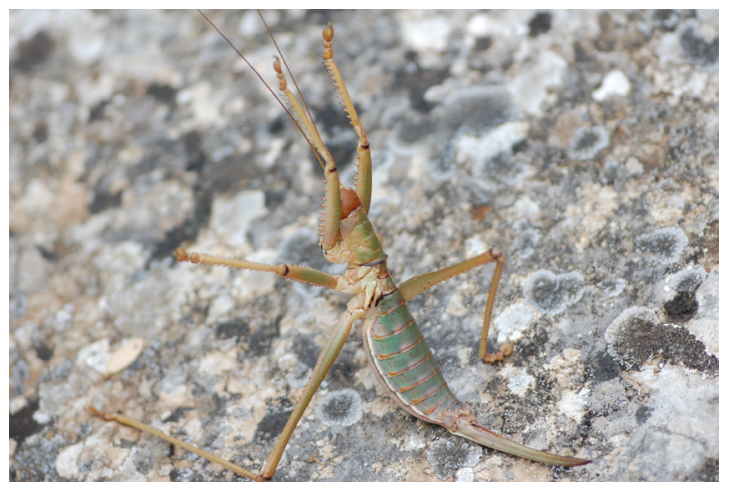

Fig. 14. Saga rhodiensis (Salfi, 1929) 


\section{Sonuç ve Tartışma}

Phaselis Antik Kenti'nde yapılmış olan Orthoptera faunası tespiti çalışmalarının ilk bölümünü içeren bu çalışmada, takımına ait, 2 alttakım, 6 familya, 15 altfamilyanın, 23 cinsine ait 27 tür/alttür tespit edilmiştir. İki alttakımdan Caelifera'ya (kısa antenli çekirgeler) ait 3 familya (Acrididae, Pamphagidae, Tetrigidae), 9 alt familya, 12 cins ve 16 tür/alttür, Ensifera'nın (uzun antenli çekirgeler) ise 3 familya (Tettigoniidae, Gryllidae, Gryllotalpidae), 6 alt familya, 11 cins ve 11 tür/alttür ile temsil edildiği ortaya çıkmıştır. Caelifera'nın Acrididae familyası 14 tür/alttür, Ensifera'nın ise Tettigoniidae familyası 7 tür/alttür ile en çok takson içeren familyalar olarak tespit edilmiştir. Bu taksonlardan tipik Akdeniz elemanı olarak Acrometopa servillea (Brullé, 1832), Bucephaloptera bucephala (Brunner von Wattenwyl, 1882) ve Calliptamus italicus italicus (Linnaeus, 1758) türleri, Afrika kökenli Eremiyal Bölge elemanı olarak Tylopsis lilifolia (Fabricius, 1793), Oecanthus pellucens pellucens (Scopoli, 1763), Aiolopus strepens strepens (Latreille, 1804), Locusta migratoria migratoria (Linnaeus, 1758), Oedipoda coerulescens (Linnaeus, 1758) ve Notostaurus anatolicus (Krauss, 1896) türleri tespit edilmiştir. Diğer taraftan Acheta domesticus (Linnaeus, 1758), Anacridium aegyptium (Linnaeus, 1764), Oedipoda aurea Uvarov, 1923, Oedipoda miniata miniata (Pallas, 1771), Gryllus (Gryllus) bimaculatus De Geer, 1773 ve Gryllotalpa gryllotalpa (Linnaeus, 1758) ise yaygın türler olarak bilinmektedir.

Çalışmada tespit edilen Poecilimon (Poecilimon) luschani birandi Karabag, 1950, türü ise Türkiye (Antalya) endemiği olarak bilinmektedir.

Diğer taraftan araştırmalar sonucunda Phaselis Antik Kenti'nde tespit edilen Caelifera türlerinden, tarım zararlısı oldukları daha önce rapor edilen ${ }^{5}$, Locusta migratoria migratoria (Linnaeus, 1758), Anacridium aegyptium (Linnaeus, 1764), Eyprepocnemis plorans plorans (Charpentier, 1825), Calliptamus italicus italicus (Linnaeus, 1758), Aiolopus strepens strepens (Latreille, 1804), Oedipoda coerulescens (Linnaeus, 1758), Notostaurus anatolicus (Krauss, 1896), Ramburiella (Palaeocesa) turcomana (Fischer von Waldheim, 1833) taksonlarıdır. Ensifera'ya ait tarım zararlıları ise; Oecanthus pellucens pellucens (Scopoli, 1763), Gryllotalpa gryllotalpa (Linnaeus, 1758) ve Tylopsis lilifolia (Fabricius, 1793) taksonlarıdır. Bu türlerden özellikle Kuzey Afrika kökenli olan Locusta migratoria migratoria dönem dönem özellikle tarımsal ve ekili alanlarda zarar oluşturabilmeleri nedeniyle müdahale edilmesi gereken popülasyonlar oluşturabilmektedir ${ }^{6}$. Ancak arazi çalışmaları yapıldığı dönemde bu taksona ait bireylerin zarar oluşturabilecek kadar yoğun bir popülasyon oluşturmadıkları tespit edilmiştir. Diğer taraftan bu türün belirli aralıklarla izlenmesi özellikle yakın çevrede yapılan tarım ve seracılık faaliyetleri için önem arz etmektedir.

Phaselis Antik Kenti'nin, sahip olduğu farklı vejetasyon tipleri ile bu çalışmada tespit edilen Orthoptera türlerinden daha fazlasını içermesi olasıdır. Alanın fiziksel olarak korunan bir alan olması, antropojenik etkinin az olması ve ziyaretçilerin belirli bir saatten sonra alanda bulunmasının yasak olmasının, özellikle gece aktif olan avcı çekirge türlerine avantaj sağladığı düşünülmektedir. Çalışma alanında ileriki yıllarda, bahar ve yaz aylarında yapılacak arazi çalışmaları ile alanın Orthoptera faunasının büyük oranda saptanacağı öngörülmektedir.

\footnotetext{
Lodos 1983; Kaya - Kovanci 2000.

6 Lodos 1983.
} 


\section{BIBBLIYOGRAFYA}

Bei-Bienko - Mistshenko 1951 G. J. Bei-Bienko - L. L. Mistshenko, The Grashopper of the Fauna of the USSR and Adjasent Countries, volume II. Moskova - Leningrad 1951.

Bei-Bienko 1965

G. J. Bei-Bienko, Fauna of USSR Orthoptera, Tettigonioidea, Phaneropterinae, Israel Program for Scientific Translation. Jerusalem 1965.

Çıplak - Demirsoy 1996

Çıplak et al. 1999

B. Çıplak - Demirsoy, "Caelifera (Orthoptera, Insecta) Altakımının Türkiye'deki Endemizm Durumu". Turkish Journal of Zoology 20 (1996) 241246.

Demirsoy 1975

B. Çıplak, B. Yalım - A. Demirsoy, Türkiye Orthoptera Faunası. 759-766. Genel ve Türkiye Zoocoğrafyası. Ankara 1999.

A. Demirsoy, Erzurum Bölgesi Orthoptera (Insecta) Faunasının Tespiti ve Taksonomik incelenmesi. Erzurum 1975.

Demirsoy 1977

Demirsoy $1999^{2}$

Eades et al. 2016

Güneş 1984

Harz 1969

Harz 1975

Karabağ 1956

Karabağ 1957

Karabağ 1958

Kaya - Kovancı 2000

Lodos 1983

Mol et al. 2014

Naskrecki - Ünal 1995

Önder et al. 1999

A. Demirsoy, Türkiye Caelifera (Insecta, Orthoptera) Faunasının Tespiti ve Taksonomik Olarak Incelenmesi. Erzurum 1977.

A. Demirsoy, Genel ve Türkiye Zoocoğrafyası: Hayvan Coğrafyası. Ankara $1999^{2}$.

D. C. Eades, D. Otte, M. M. Cigliano - H. Braun, Orthoptera Species File. Version 5.0/5.0. February 2016. Source: http://orthoptera. speciesfile.org. H. V. Güneş, Doğu Akdeniz Bölgesi Orthoptera (Insecta) Faunası Üzerine Taksonomik Çalışmalar. Yayımlanmamış Doktora Tezi, Ankara Üniversitesi. Ankara 1984.

K. Harz, The Orthoptera of Europe, volume I. The Hauge 1969.

K. Harz, The Orthoptera of Europe, volume II. The Hauge 1975.

T. Karabağ, "Some New and Less Known Tettigoniidae (Orthoptora) from Turkey". Comm. Fac. Sci. Univ. Ankara 5 (1956) 1-19.

T. Karabağ, "Some New Orthoptera from Turkey". Comm. Fac. Sci. Univ. Ankara 8 (1957) 13-18.

T. Karabağ, Türkiye'nin Orthoptera Faunası. İstanbul 1958.

M. Kaya - B. Kovancı, "Bursa'da Saptanan Ahududu Zararlıları". Ed. Anonymous, Türkiye 4. Entomoloji Kongresi Bildirileri. Aydın (2000) 455465.

N. Lodos, Türkiye Entomolojisi I (Genel Uygulamalı ve Faunistik). İzmir 1983.

A. Mol, D. Şirin - M. S. Taylan, "Türkiye'de Dağılım Gösteren Bazı Caelifera (Insecta: Orthoptera) Türlerinin Yeni Lokalite Kayıtları, Endemizm, Yaygınlık ve Tarımsal Zarar Oluşturma Açısından Değerlendirilmesi”. Bitki Koruma Bülteni 54/2 (2014) 133-170.

P. Naskrecki - M. Ünal, "The Orthoptera of Hatay Province, S. Turkey". Bietrage Entomologia 45 (1995) 393-420.

F. Önder, E. Pehlivan, Y. Karsavuran, T. Serdar - Ş. Kısmalı, "Catalogue of Collection of Pamphagidae, Pyrgomorphidae, Catantopidae and Acrididae (Orthoptera: Acridoidea) Preserved in the Prof. Dr. Niyazi LODOS Museum, İzmir, Turkey". Türk Entomologi Dergisi 23 (1999) 163-178.

Ramme 1939 W. Ramme, "Beitrage zur Kenntis der palaearktischen Orhopterenfauna (Tettig. u. Acrid.)". Mitteilungen aus dem Zoology Museum Berlin 24 (1939) 41-150.

Ramme 1951

W. Ramme. "Zur systematik, Faunistik und Biologie der Orthopteren von Südost Europa Und Vorderasien". Zoological Museum Berlin 27 (1951) 1431.

Salman 1978 
56

Salman 1981

Sevgili et al. 2011

Soltani 1978

Şirin et al. 2010

Tazegül - Önder 2012

Ünal 1999

Ünal 2001

Ünal 2011

Uvarov 1934

Weidner 1969

Willemse 1984

Willemse 1985

Yalım - Çıplak 2002

\section{Mehmet Sait TAYLAN - Deniz ŞiRiN}

Üzerine Taksonomik Araştırmalar. Erzurum 1978.

S. Salman, Güney ve Güney Doğu Anadolu'da Pholidopterini (Orthoptera, Decticinae) Tribusu Üzerine Taksonomik Araştırmalar. Erzurum 1981.

H. Sevgili, A. Demirsoy - Y. Durmuş, "Orthoptera and Mantodea Fauna of Kazdağı (Ida) National Park with Data on the Calling Songs of Some BushCrickets". Turkish Journal of Zoology 35 (2011) 631-652.

A. A. Soltani, "Preliminary Synonym and Description of New Species in the Genus Dociostaurus Fieber, 1853 (Orthoptera: Acridoidea; Acrididae, Gomphocerinae) with a Key to the Species in the Genus". Journal of African Ent. Society of Iran Suppl 2 (1978) 1-93.

D. Şirin, O. Eren - B. Çıplak, "Grasshopper Diversity and Abundance in Relation to Elevation and Vejetation from a Snapshot in Mediterranean Anatolia: Role of Latitudinal Position in Altitudinal Differences". Journal of Natural History 44 (2010) 1343-1363.

E. Tazegül - F. Önder, "İzmir Ilinde Bulunan Tettigoniidae (Orthoptera) Familyası Türleri Üzerinde Sistematik Araştırmalar”. Türk. Entomol. Bült. 2 (2012) 109-123.

M. Ünal, "Notes on Orthoptera of Western Turkey, with Description of a New Genus and Four New Species". Journal of Orthoptera Research 8 (1999) 243-255.

M. Ünal, "Contributions to the Orthoptera Fauna of the Middle Taurus Mountains (South Turkey)". Miscellaneous Papers 76 (2001) 1-8.

M. Ünal, Turkish Orthoptera Site (TOS). Source: www.orthoptera-tr.org (retrieval at February 2016).

B. P. Uvarov, "Studies in the Orthoptera of Turkey, Iraq and Syria". Enstituto Espanol de Entomologia 10 (1934) 21-119.

H. Weidner, "Beitrage zur Kenntnis der Feldheuschenrecken (Caelifera) Anatoliens". Mittellungen aus dem Zoology Museum of Berlin 66 (1969) 145-226.

F. Willemse, Catalogue of the Orthoptera of Greece I. Athens 1984.

F. Willemse, Fauna Graeciae II. A Key to the Orthoptera Species of Greece. Athens 1985.

B. Yalım - B. Çıplak, "Termessos Milli Parkı (Antalya) Orthoptera (Insecta) Faunası: Fauna Elemanlarının Zoocoğrafyaları ve Vejetasyona Göre Dağılışları". Türkiye Entomoloji Dergisi 26 (2002) 267-276. 